\title{
A Call for a New Paradigm for Diabetes Care in the Era of Sodium-Glucose Cotransporter 2 Inhibitors (SGLT2i)
}

\author{
Sun H. Kim (D) - Tara I. Chang • Kenneth W. Mahaffey
}

Received: June 4, 2020 / Published online: July 13, 2020

(C) The Author(s) 2020

\section{ABSTRACT}

In 2013, canagliflozin was the first sodiumglucose cotransporter 2 inhibitor (SGLT2i) approved by the US Food and Drug Administration for the treatment of type 2 diabetes (T2DM). Today, there are four SGLT2i approved for T2DM, and some SGLT2i have been approved for indications beyond glucose control. For example, SGLT2i reduce major adverse clinical events (MACE) including nonfatal

Digital Features To view digital features for this article go to https://doi.org/10.6084/m9.figshare.12600092.

\section{S. H. Kim (ه)}

Division of Endocrinology, Gerontology and Metabolism, Department of Medicine, Stanford University School of Medicine, Stanford, CA, USA e-mail: sunhkim@stanford.edu

\section{T. I. Chang}

Division of Nephrology, Department of Medicine, Stanford University School of Medicine, Stanford, CA, USA

\section{K. W. Mahaffey}

Stanford Center for Clinical Research, Department of Medicine, Stanford University School of Medicine, Stanford, CA, USA

S. H. Kim - T. I. Chang - K. W. Mahaffey Stanford Diabetes Research Center, Stanford University School of Medicine, Stanford, CA, USA myocardial infarction, nonfatal stroke, and cardiovascular death (canagliflozin); cardiovascular death (empagliflozin, dapagliflozin); diabetic kidney disease progression (canagliflozin); and heart failure hospitalization (canagliflozin, dapagliflozin). However, despite the potential benefits of SGLT2i in reducing adverse clinical events, providers underprescribe SGLT2i for eligible patients. Thus, we propose the CKD-PCP framework which allows multiple providers to utilize the benefits of SGLT2i. CKD-PCP has dual meaning: it applies to providers who most often care for patients with T2DM (Cardiologists, Kidney specialists, Diabetologists, and Primary Care Physicians) and it refers to the benefits of SGLT2i (treatment of Cardiovascular disease, Kidney disease, Diabetes, and reduction of blood Pressure, Calories, and Plasma volume). This article is based on previously conducted studies and the authors disclose their roles in relevant trials in the Acknowledgements.

Keywords: Heart failure; Kidney disease; Proteinuria; SGLT2 inhibitors; Type 2 diabetes 


\section{Key Summary Points}

Sodium-glucose cotransporter 2 inhibitors (SGLT2i) are approved for the treatment of type 2 diabetes.

On the basis of large clinical trials, some SGLT2i also have indications beyond glucose control. For example, SGLT2i reduce major adverse clinical events (MACE) including nonfatal myocardial infarction, nonfatal stroke, and cardiovascular death (canagliflozin); cardiovascular death (empagliflozin, dapagliflozin); diabetic kidney disease progression (canagliflozin); and heart failure hospitalization (canagliflozin, dapagliflozin).

Real-world evidence shows underutilization of SGLT2i for eligible patients.

To optimize use of SGLT2i, we propose a new team-based framework, CKD-PCP, which has dual meaning: it applies to providers who most often care for patients with T2DM (Cardiologists, Kidney specialists, Diabetologists, and Primary Care Physicians) and it refers to the benefits of SGLT2i (treatment of Cardiovascular disease, Kidney disease, Diabetes, and reduction of blood Pressure, Calories, and Plasma volume).

Approximately $12 \%$ of US adults have type 2 diabetes (T2DM). Persons with T2DM commonly have comorbid conditions and are at high risk for micro- and macrovascular complications. Results from several large clinical trials (Table 1) of patients with T2DM who are treated with sodium-glucose cotransporter 2 inhibitors (SGLT2i) have generated intense interest because this class of medications provides benefits beyond glucose control such as a reduction in cardiovascular events and a slower progression of kidney disease in patients with T2DM [1-5].
Since 2008, the US Food and Drug Administration (FDA) has mandated that new drugs for T2DM demonstrate cardiovascular safety and noninferiority to placebo treatment. In 2015, the first cardiovascular outcome trial with an SGLT2i, empagliflozin, unexpectedly revealed that treatment with empagliflozin was superior to placebo in the EMPA-REG OUTCOME trial (Empagliflozin Cardiovascular Outcome Event Trial in Type 2 Diabetes-Removing Excess Glucose [1], Table 1). Specifically, patients with T2DM treated with empagliflozin had a $14 \%$ decreased risk of major adverse clinical events (MACE) including nonfatal myocardial infarction, nonfatal stroke, and cardiovascular death compared to those on placebo [1]. In 2017, patients with T2DM treated with canagliflozin also had a similar 14\% reduction in MACE in CANVAS (Canagliflozin Cardiovascular Assessment Study) [2]. Outcome trials of dapagliflozin [5] and ertugliflozin [6] showed noninferiority, though not superiority, in MACE when compared to placebo (Table 1 ).

While initial trials of SGLT2i were needed to demonstrate cardiovascular safety, two recent SGLT2i trials were conducted to evaluate benefits of SGLT2i in patients with diabetic kidney disease [4] and history of heart failure [3]. The CREDENCE (Canagliflozin and Renal Events in Diabetes with Established Nephropathy Clinical Evaluation) trial was the first study dedicated to the evaluation of kidney outcomes in a population with T2DM and albuminuric diabetic kidney disease (urine albumin-to-creatinine ratio, UACR, > $300 \mathrm{mg} / \mathrm{g}$ ) [4]. Kidney outcomes included progression to end-stage kidney disease (dialysis, transplantation, or a sustained estimated glomerular filtration rate $($ eGFR $)<15 \mathrm{~mL} / \mathrm{min} / 1.73 \mathrm{~m}^{2}$ ), a doubling of the serum creatinine level, or death from renal or cardiovascular causes. The trial was stopped early owing to benefit and showed that treatment with canagliflozin reduced kidney outcomes by $30 \%$ compared to placebo. In addition, CREDENCE confirmed cardiovascular benefits seen in CANVAS [2] and showed a 20\% reduction in MACE in patients treated with canagliflozin versus placebo [2]. These results are particularly striking when considering that the benefit of canagliflozin was apparent in 
Table 1 Current SGLT2i approved for treatment of T2DM and pivotal trial data

\begin{tabular}{|c|c|c|c|c|}
\hline $\begin{array}{l}\text { FDA } \\
\text { approved } \\
\text { SGLT2i }\end{array}$ & $\begin{array}{l}\text { Pivotal } \\
\text { clinical trial } \\
(N)\end{array}$ & Main inclusion criteria & $\begin{array}{l}\text { Primary outcome (HR, } 95 \% \\
\text { CI) }\end{array}$ & $\begin{array}{l}\text { Additional FDA } \\
\text { indication }\end{array}$ \\
\hline \multirow[t]{2}{*}{ Canagliflozin } & $\begin{array}{r}\text { CANVAS [2] } \\
\qquad N=10,142\end{array}$ & $\begin{array}{l}\text { T2DM and established } \\
\text { ASCVD at } \geq 30 \text { years of } \\
\text { age or }>2 \text { ASCVD risk } \\
\text { factors at } \geq 50 \text { years of } \\
\text { age }\end{array}$ & $\begin{array}{l}\text { 3-point MACE; } 0.86 \\
\quad(0.75-0.97)\end{array}$ & $\downarrow \mathrm{MACE}$ \\
\hline & $\begin{array}{l}\text { CREDENCE } \\
\text { [4] } \\
\quad N=4401\end{array}$ & $\begin{array}{l}\text { T2DM and eGFR } \\
30-90 \mathrm{~mL} / \mathrm{min} / 1.73 \mathrm{~m}^{2} \\
\text { and UACR } \\
300-5000 \mathrm{mg} / \mathrm{g}\end{array}$ & $\begin{array}{l}\text { Composite ESKD, doubling of } \\
\text { serum creatinine, or death } \\
\text { from renal or cardiovascular } \\
\text { causes; } 0.70(0.59-0.82)\end{array}$ & $\begin{array}{l}\downarrow \text { Diabetic kidney disease, } \\
\downarrow \text { heart failure } \\
\text { hospitalization }\end{array}$ \\
\hline Empagliflozin & $\begin{array}{l}\text { EMPA-REG } \\
\qquad \begin{array}{l}{[1]} \\
N=7020\end{array}\end{array}$ & $\begin{array}{l}\text { T2DM and preexisting } \\
\text { ASCVD }\end{array}$ & $\begin{array}{l}\text { 3-point MACE; } 0.86 \\
\quad(0.74-0.99)\end{array}$ & $\downarrow$ Cardiovascular death \\
\hline \multirow[t]{3}{*}{ Dapagliflozin } & $\begin{array}{l}\text { DECLARE- } \\
\text { TIMI [5], } \\
N=17,160\end{array}$ & $\begin{array}{l}\text { T2DM and established } \\
\text { ASCVD or multiple risk } \\
\text { factors }\end{array}$ & $\begin{array}{l}\text { 3-point MACE; } 0.93 \\
\quad(0.84-1.03)\end{array}$ & $\begin{array}{l}\downarrow \text { Heart failure } \\
\quad \text { hospitalization }\end{array}$ \\
\hline & $\begin{array}{l}\text { DAPA-HF } \\
\qquad \begin{array}{l}{[3]} \\
N=4744\end{array}\end{array}$ & $\begin{array}{l}\text { Left ventricular ejection } \\
\text { fraction } \leq 40 \% ; 42 \% \\
\text { with T2DM }\end{array}$ & $\begin{array}{l}\text { Composite of worsening heart } \\
\text { failure or CV death; } 0.74 \\
(0.65-0.85)\end{array}$ & $\begin{array}{l}\downarrow \text { Heart failure with } \\
\text { reduced ejection } \\
\text { fraction (HFrEF) with } \\
\text { and without T2DM, } \\
\downarrow \text { CV death }\end{array}$ \\
\hline & $\begin{array}{c}\text { DAPA-CKD, } \\
N=4245\end{array}$ & $\begin{array}{l}\text { eGFR } 25-75 \mathrm{~mL} / \mathrm{min} / \\
1.73 \mathrm{~m}^{2} \text { and } \\
\mathrm{UACR} \geq 200 \mathrm{mg} / \\
\mathrm{g} ; \sim 70 \% \text { with T2DM }\end{array}$ & $\begin{array}{l}\text { Composite of eGFR } \geq 50 \% \text {, } \\
\text { ESKD, renal death or } \mathrm{CV} \\
\text { death }\end{array}$ & $\begin{array}{l}\text { (Terminated early for } \\
\text { benefit) }\end{array}$ \\
\hline Ertugliflozin & $\begin{array}{l}\text { VERTIS-CV, } \\
\qquad N=8246\end{array}$ & $\begin{array}{l}\text { T2DM and established } \\
\text { ASCVD }\end{array}$ & $\begin{array}{l}\text { 3-point MACE } \\
0.97(0.85-1.11)\end{array}$ & $\begin{array}{l}\text { Showed non-inferiority } \\
\text { but not superiority on } \\
\text { MACE }\end{array}$ \\
\hline
\end{tabular}

Pending Trials: EMPEROR-REDUCED (Empagliflozin Outcome Trial in Patients with Chronic Heart Failure with Reduced Ejection Fraction). EMPEROR-PRESERVED (Empagliflozin Outcome Trial in Patients with Chronic Heart Failure with Preserved Ejection Fraction). EMPA-KIDNEY (The Study of Heart and Kidney Protection with Empagliflozin). DELIVER (Dapagliflozin Evaluation to Improve the Lives of Patients with Preserved Ejection Fraction Heart Failure)

ASCVD atherosclerotic cardiovascular disease, 3-point $M A C E$ major adverse cardiovascular events including nonfatal myocardial infarction, nonfatal stroke, and cardiovascular death, $e G F R$ estimated glomerular filtration rate, ESKD end-stage kidney disease, $U A C R$ urine albumin-to-creatinine ratio

patients on maximally tolerated dose of angiotensin-converting enzyme inhibitors (ACEi) or angiotensin II receptor blockers (ARB). On the basis of these results, the FDA approved a new indication for canagliflozin for the treatment of diabetic kidney disease in September 2019, 
marking the first drug since ACEi and ARBs to demonstrate renoprotective benefits in patients with diabetic kidney disease.

The DAPA-CKD (Dapagliflozin And Prevention of Adverse outcomes in CKD) study is the second trial dedicated to kidney-disease-related outcomes in patients with chronic kidney disease (eGFR 25-75 mL/min/1.73 $\mathrm{m}^{2}$ and UACR $\geq 200 \mathrm{mg} / \mathrm{g}$ ) and in those with and without T2DM [7]. Like CREDENCE [4], the primary outcome includes a composite kidney outcome ( $\geq 50 \%$ sustained decline in eGFR, end-stage kidney disease) or renal or cardiovascular death. The DAPA-CKD trial terminated early in March 2020 for benefits in the dapagliflozin group; however, full results have not been released. The EMPA-KIDNEY (The Study of Heart and Kidney Protection with Empagliflozin) study is ongoing and evaluates the effects of empagliflozin for patients with chronic kidney disease (eGFR 20-45 or $45-90 \mathrm{~mL} / \mathrm{min} / 1.73 \mathrm{~m}^{2}$ with UACR $\geq 200 \mathrm{mg} /$ g) on composite kidney outcomes (end-stage kidney disease, sustained decline in eGFR $<10 \mathrm{~mL} / \mathrm{min} / 1.73 \mathrm{~m}^{2}$, sustained decline of $\geq 40 \%$ in eGFR from randomization) or renal or cardiovascular death.

The DAPA-HF (Dapagliflozin and Prevention of Adverse Outcomes in Heart Failure) trial was the first SGLT2i study to enroll participants with reduced ejection fraction $\leq 40 \%$ with and without T2DM [3]. The DAPA-HF cohort included $42 \%$ of subjects with baseline T2DM; the results showed that dapagliflozin reduced a composite of worsening heart failure (hospitalization or an urgent visit resulting in intravenous therapy for heart failure) or cardiovascular death by $26 \%$. This trial not only demonstrated benefits of SGLT2i for heart failure and cardiovascular death but importantly it showed similar efficacy for individuals with and without T2DM. In addition, SGLT2i showed cardiac benefits for patients who were already on standard therapy for heart failure which included ACEi/ARBs or sacubitril-valsartan, beta-blockers, and mineralocorticoid receptor antagonists. Ongoing trials that are evaluating primary heart failure outcomes include dapagliflozin in patients with preserved ejection fraction (DELIVER) and empagliflozin in patients with reduced (ejection fraction $\leq 40 \%$ ) and preserved ejection fraction (EMPEROR-Reduced and EMPEROR-Preserved, respectively).

Several mechanisms have been proposed to underlie the cardiovascular and renal benefits of SGLT2i. Foremost, SGLT2i inhibit renal reabsorption of glucose, as well as sodium, in the proximal renal tubules, leading to glycosuria and natriuresis [8]. These direct effects of SGLT2i explain the decrease in serum glucose, weight, and blood pressure in patients who are treated with SGLT2i. In addition, SGLT2i also have indirect hemodynamic and metabolic effects. Inhibition of proximal sodium reabsorption leads to increased delivery of sodium to the macula densa, which stimulates tubuloglomerular feedback and afferent arterial vasoconstriction, and reduces glomerular hyperfiltration [9]. This cascade is thought to play a major role in the renoprotective effects of SGLT2i. SGLT2i also alter the insulin to glucagon ratio and increase hepatic ketone production which are believed to be efficient fuel sources for cardiomyocytes [10] and may mediate the cardiac benefits of SGLT2i. Lastly, SGLT2i may also inhibit sodium-hydrogen exchangers in the heart, which may protect against sodium overload in a failing cardiomyocyte and improve calcium availability in the mitochondria, which are needed for energy generation [11].

We now have compelling evidence to start SGLT2i in patients with T2DM and kidney disease or heart failure. In the 2020 Standards of Medical Care in Diabetes [12], the American Diabetes Association (ADA) recommends SGLT2i for patients with T2DM and chronic kidney disease (defined as an eGFR 30-60 mL/ $\mathrm{min} / 1.73 \mathrm{~m}^{2}$ or UACR $>30 \mathrm{mg} / \mathrm{g}$, particularly $>300 \mathrm{mg} / \mathrm{g}$ ) on the basis of findings from the CREDENCE study [4]. Additionally, the ADA recommends SGLT2i for patients with heart failure (particularly, left ventricular ejection fraction $<45 \%$ ) on the basis of findings from DAPA-HF [3]. Finally, the ADA recommends treatment of patients with an SGLT2i regardless of baseline hemoglobin A1c or individualized A1c target to reduce diabetic kidney disease progression, hospitalization for heart failure, MACE, and/or cardiovascular death. The ADA 


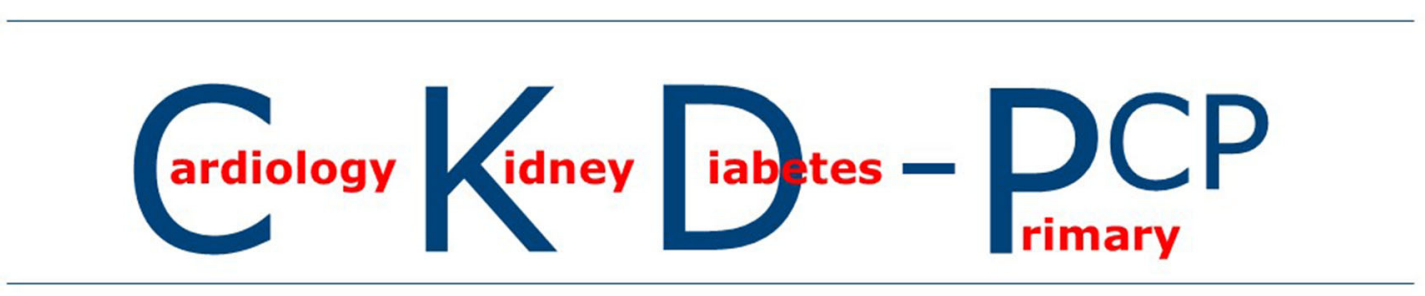

\section{SGLT2i for: Treatment of Cardiovascular disease and heart failure \\ Treatment of diabetic Kidney disease \\ Treatment of Diabetes}

Reduction in blood Pressure, Calories, Plasma volume, and more.

Fig. 1 CKD-PCP: Team-based care for T2DM representing involved providers and benefits of SGLT2i

continues to recommend SGLT2i after metformin and lifestyle changes regardless of comorbidities, although the European Society of Cardiology suggests SGLT2i as first-line treatment for those with or those at high or very high risk for atherosclerotic cardiovascular disease [13]. High risk for atherosclerotic cardiovascular disease is defined as duration of diabetes for at least 10 years with any other risk factor (age, hypertension, dyslipidemia, smoking, or obesity). Very high risk is defined as three or more risk factors or organ damage (proteinuria, eGFR $<30 \mathrm{~mL} / \mathrm{min} / 1.73 \mathrm{~m}^{2}$, left ventricular hypertrophy, or retinopathy).

How do we implement these new recommendations to patients with T2DM? The pleiotropic benefits of SGLT2i presents a happy conundrum for providers who care for patients with T2DM with multiple comorbid conditions. Some patients with T2DM may see several care specialists, including endocrinologists, nephrologists, and cardiologists, but a majority of these patients may only see their primary care physician. We believe any provider caring for a patient with T2DM should consider prescribing an SGLT2i, but these medications do require careful monitoring for adverse effects by both the patient and provider. SGLT2i have been associated with significant risk for genital mycotic infections, particularly in women with previous episodes of genital infections and uncircumcised men. In addition, although rare, SGLT2i have been associated with increased risk for necrotizing fasciitis of the perineum or Fournier's gangrene. SGLT2i also increase risk for hypoglycemia in patients on concurrent sulfonylureas and insulin and pose risk for diabetic ketoacidosis during stress (e.g., perioperative setting or intercurrent illness with reduced food and fluid intake and reduced insulin doses). Providers should also be aware that SGLT2i treatment can lead to an acute fall in eGFR that is similar to that observed with initiation of ACEi and ARB. However, this limited fall in eGFR in the short term is not a side effect, per se, but instead reflects a decrease in hyperfiltration that will ultimately slow progression of kidney disease in the long term. Finally, canagliflozin may increase the risk for amputations on the basis of findings in CANVAS [2] but not in CREDENCE [4]. Thus, providers cannot casually initiate SGLT2i without discussing risks, monitoring for complications, or adjusting medications.

Although team-based or patient-centered care is not a new concept, it is an unfamiliar approach in the new era of diabetes care with the SGLT2i. We now have an intervention that improves the metabolic profile, reduces cardiovascular outcomes, and delays the progression of kidney failure for patients with T2DM and diabetic kidney disease. In our health care systems, we need to empower all clinical care groups to consider therapy with an SGLT2i, if indicated, rather than wait or defer a decision to start SGLT2i to one of the other specialists or primary care physician.

Real-world evidence demonstrates that SGLT2i are currently underutilized for eligible patients $[14,15]$ and that the decision to start 
SGLT2i is typically deferred to the endocrinologist [15]. Thus, we propose a new decision framework, CKD-PCP (Fig. 1), that encourages each provider to focus on the key area of interest yet ensures that the metabolic profile, cardiovascular and kidney outcomes are comprehensively addressed. CKD-PCP thus has dual meaning: it applies to providers who most often care for patients with T2DM (Cardiologists, Kidney specialists, Diabetologists, and Primary Care Physicians) and it refers to the benefits of SGLT2i (treatment of Cardiovascular disease, Kidney disease, Diabetes, and reduction of blood Pressure, Calories, and Plasma volume).

Without head-to-head comparisons between the four commercially available SGLT2i, the selection of a particular agent in this class remains at the discretion of the provider. Data is currently available for the benefits of SGLT2i in patients with T2DM and kidney disease (canagliflozin) or patients without diabetes and heart failure (dapagliflozin). We now await the full results DAPA-CKD to understand how dapagliflozin benefits the kidney function of patients with and without T2DM.

\section{ACKNOWLEDGEMENTS}

Funding. There was no funding for this commentary. No Rapid Service Fee was received by the journal for the publication of this article.

Authorship. All named authors meet the International Committee of Medical Journal Editors (ICMJE) criteria for authorship for this article, take responsibility for the integrity of the work as a whole, and have given their approval for this version to be published.

Disclosures. Sun H Kim was a site investigator for the CREDENCE study. Tara I Chang was on the events adjudication committee and a US National Leader for CREDENCE. Kenneth W Mahaffey was a co-chair of the CREDENCE steering committee and was also on the CANVAS steering committee.
Compliance with Ethics Guidelines. This article is based on previously conducted studies and does not contain any studies with human participants or animals performed by any of the authors except as noted in Disclosures.

Prior presentation. Information was presented at the Stanford Department of Medicine Grand Rounds on May 29, 2019.

Data Availability. Data sharing is not applicable to this article as no datasets were generated or analyzed during the current study.

Open Access. This article is licensed under a Creative Commons Attribution-NonCommercial 4.0 International License, which permits any non-commercial use, sharing, adaptation, distribution and reproduction in any medium or format, as long as you give appropriate credit to the original author(s) and the source, provide a link to the Creative Commons licence, and indicate if changes were made. The images or other third party material in this article are included in the article's Creative Commons licence, unless indicated otherwise in a credit line to the material. If material is not included in the article's Creative Commons licence and your intended use is not permitted by statutory regulation or exceeds the permitted use, you will need to obtain permission directly from the copyright holder. To view a copy of this licence, visit http:// creativecommons.org/licenses/by-nc/4.0/.

\section{REFERENCES}

1. Zinman B, Wanner C, Lachin JM, et al. Empagliflozin, cardiovascular outcomes, and mortality in type 2 diabetes. N Engl J Med. 2015;373(22): 2117-288.

2. Neal B, Perkovic V, Mahaffey KW, et al. Canagliflozin and cardiovascular and renal events in type 2 diabetes. N Engl J Med. 2017;377(7):644-57.

3. McMurray JJV, Solomon SD, Inzucchi SE, et al. Dapagliflozin in patients with heart failure and reduced ejection fraction. $\mathrm{N}$ Engl $\mathrm{J}$ Med. 2019;381(21):1995-2008. 
4. Perkovic V, Jardine MJ, Neal B, et al. Canagliflozin and renal outcomes in type 2 diabetes and nephropathy. N Engl J Med. 2019;380(24): 2295-306.

5. Wiviott SD, Raz I, Bonaca MP, et al. Dapagliflozin and cardiovascular outcomes in type 2 diabetes. N Engl J Med. 2019;380(4):347-57.

6. Cannon C. Cardiovascular outcomes following ertugliflozin treatment in patients with type 2 diabetes mellitus and atherosclerotic cardiovascular disease (VERTIS CV Trial). American Diabetes Association 80th Scientific Sessions. Virtual 2020.

7. Heerspink HJL, Stefansson BV, Chertow GM, et al. Rationale and protocol of the dapagliflozin and prevention of adverse outcomes in chronic kidney disease (DAPA-CKD) randomized controlled trial. Nephrol Dial Transplant. 2020;35(2):274-82.

8. Ghezzi C, Loo DDF, Wright EM. Physiology of renal glucose handling via SGLT1, SGLT2 and GLUT2. Diabetologia. 2018;61(10):2087-97.

9. Fioretto P, Zambon A, Rossato M, Busetto L, Vettor R. SGLT2 inhibitors and the diabetic kidney. Diabetes Care. 2016;39(Suppl 2):S165-S171171.

10. Santos-Gallego CG, Requena-Ibanez JA, San Antonio R, et al. Empagliflozin ameliorates adverse left ventricular remodeling in nondiabetic heart failure by enhancing myocardial energetics. J Am Coll Cardiol. 2019;73(15):1931-44.

11. Arjun S, Bell RM. SGLT2 inhibitors: reviving the sodium-hydrogen exchanger cardioprotection hypothesis? Cardiovasc Res. 2019;115(10):1454-6.

12. American Diabetes Association. 9. Pharmacologic approaches to glycemic treatment: Standards of Medical Care in Diabetes-2020. Diabetes Care. 2020;43(Suppl 1):S98-S110.

13. Cosentino F, Grant PJ, Aboyans V, et al. 2019 ESC Guidelines on diabetes, pre-diabetes, and cardiovascular diseases developed in collaboration with the EASD: The Task Force for diabetes, pre-diabetes, and cardiovascular diseases of the European Society of Cardiology (ESC) and the European Association for the Study of Diabetes (EASD). Eur Heart J. 2020;41:255-323.

14. Arnold SV, Inzucchi SE, Tang F, et al. Real-world use and modeled impact of glucose-lowering therapies evaluated in recent cardiovascular outcomes trials: an NCDR R Research to Practice project. Eur J Prev Cardiol. 2017;24(15):1637-45.

15. Vaduganathan M, Sathiyakumar V, Singh A, et al. Prescriber patterns of SGLT2i after expansions of US food and drug administration labeling. J Am Coll Cardiol. 2018;72(25):3370-2. 